\title{
Crossmodal Interaction: Using Audio or Tactile Displays in Mobile Devices
}

\author{
Eve Hoggan \\ Multimodal Interaction Group I GIST, University of Glasgow, UK, G12 8QQ \\ eve@dcs.gla.ac.uk
}

\begin{abstract}
Mobile device users can be in a variety of different situations where visual, audio, or tactile feedback is not appropriate. This research aims to investigate the design of auditory/tactile crossmodal icons which can provide an alternative form of output using the most appropriate modality to communicate information. The results of this research will aid designers of mobile displays in creating effective crossmodal cues which require minimal training and provide alternative presentation modalities through which information may be presented if the context requires.
\end{abstract}

\section{Introduction}

Providing non-visual information to mobile users is an important area of research. We spend a great deal of our lives using mobile devices. Whether it is in a bag or we are at a noisy party, we still want to be able to interact with our device. In these situations, visual feedback is not always appropriate. However, although a user's eyes may be busy focusing on their primary task, many activities do not otherwise restrict users from attending to information using their other senses.

Manufacturers already include basic audio and vibrotactile features in products like mobile phones. Unfortunately, when the device is in a bag or pocket, tactile feedback can go unnoticed. If a user is listening to music, audio feedback can be ineffective. Thus, mobile applications could benefit from providing alternative presentation modalities depending on the situation. As the context changes, so should the feedback modality. This research exploits existing features of mobile devices by making information available to both the auditory and tactile senses so that the user can receive the information in the most suitable way.

\section{Crossmodal Auditory and Tactile Interaction}

This research investigates the design of crossmodal auditory and tactile feedback using crossmodal icons [4]. Crossmodal icons can be automatically instantiated as an Earcon [1] or Tacton [2], such that the resultant cues are equivalent and can be compared as such; enabling mobile devices to output the same information interchangeably via different modalities. 
The crossmodal parameters used in auditory and tactile icons to encode the same information are the amodal attributes available in those two senses e.g. intensity, rate, rhythmic structure, texture and spatial location. The first stage in this research was to identify the different crossmodal parameters which could be used as feedback and what sort of information could be encoded in such parameters.

\subsection{Crossmodal Parameter Experiments and Application of Parameters}

Three experiments investigating possible parameters and mappings have been conducted so far to determine which audio or tactile parameters can be considered as amodal and can map the same information between modalities. This is difficult because some of the most effective parameters available in the audio domain do not have direct mappings to the tactile domain and vice versa. So far, the experiments have investigated rhythms with texture and spatial location as potential parameters in both stationary and mobile environments [3, 4]. Results show that mapping audio with differing timbres or amplitude modulation to tactile amplitude modulation can create crossmodal roughness and 3D audio spatial locations can be mapped to tactile body positions on the waist with $72 \%$ accuracy when the user is mobile.

A complete set of crossmodal icons have now been created which use a combination of these parameters to encode information across the modalities. The experiment underway at present is investigating whether, if a user is trained to understand 3 dimensional alerts in one modality, they can then identify them in the other. The results for overall crossmodal Earcon recognition when trained with crossmodal Tactons show an average recognition rate of $85.1 \%$ while the results for overall crossmodal Tacton recognition when trained with crossmodal Earcons show rates of $75 \%$. Early results are positive and indicate that once trained in one modality users can then understand the crossmodal cues in the other modality.

\section{Future Work}

Now that we have a complete set of 3 dimensional crossmodal icons we intend to address several research questions. What types of applications would benefit from crossmodal icons? Can crossmodal feedback aid users in mobile touchscreen interaction, navigation and collaborative tasks? What methods can be used to teach users to understand crossmodal alerts? What habits do mobile users develop? If users are given a choice of modalities, which combination do they choose to use, in what applications and in what situations? In order to answer these questions our research will involve integrating and evaluating crossmodal icons in several mobile applications which allow for the varying physical and social environments within which such devices are used.

Our research has shown that feedback can be created which exploits users' abilities to transfer knowledge from one modality to another By taking this into account and designing applications with adaptive crossmodal feedback, users will have the ability to interact with their devices even when their situation and surroundings change. 


\section{References}

1. Blattner, M.M., Sumikawa, D.A., Greenberg, R.M.: Earcons and Icons: Their Structure and Common Design Principles. Human Computer Interaction 4(1), 11-44 (1989)

2. Brown, L.M., Brewster, S.A., Purchase, H.C.: A First Investigation into the Effectiveness of Tactons. In: Proc WorldHaptics 2005, pp. 167-176. IEEE, Los Alamitos (2005)

3. Hoggan, E., Brewster, S.: Crossmodal Icons for Information Display. In: Proc CHI '06 Extended Abstracts, pp. 857-862. ACM Press, New York (2006)

4. Hoggan, E., Brewster, S.A.: Crossmodal Spatial Location: Initial Experiments. In: Proc NordiCHI, Norway, pp. 469-472. ACM, New York (2006) 